\section{EL BUEN GOBIERNO DE LOS ROBOTS PARA SU CORRECTO IMPACTO EN LA SOCIEDAD: ¿QUÉ HERRAMIENTAS EXISTEN?}

\author{
Christi Amesti Mendizábal \\ Universidad Complutense de Madrid \\ https://orcid.org/0000-0002-2304-3121 \\ camestim@ucm.es \\ Natalia Zardoya Jiménez \\ https://orcid.org/0000-0002-4168-4571 \\ nataliazardoyaj@hotmail.com
}

\begin{abstract}
Cómo citar este artículo/Citation: Amesti Mendizábal, Christi; Zardoya Jiménez, Natalia (2021). El buen gobierno de los robots para su correcto impacto en la sociedad: ¿qué herramientas existen?. Arbor, 197(802): a625. https://doi.org/10.3989/arbor.2021.802003

Recibido: 1 marzo 2021. Aceptado: 22 octubre 2021

Publicado: 3 febrero 2022

RESUMEN: La inteligencia artificial (IA) y la robótica son dos realidades que han venido para quedarse y cuya implicación en nuestra sociedad afecta a distintos ámbitos. El hecho de que nos encontremos ante una tecnología capaz de emular actitudes humanas como son el aprendizaje o la adaptación, convierten a la IA, y en concreto a los robots dotados de la misma, en máquinas imprevisibles. Asimismo, nos encontramos con la agravante de la posible interacción entre máquinas y sujetos de carácter vulnerable como son los consumidores o los pacientes, lo que entraña un riesgo aún mayor. Precisamente por ello es necesario un análisis desde el punto de vista jurídico, así como también ético, de las consecuencias del manejo de los robots inteligentes en nuestra sociedad.
\end{abstract}

PALABRAS CLAVE: Robótica, inteligencia artificial, responsabilidad, protección de datos, regulación, COVID-19.

\section{GOVERNANCE OF ROBOTS FOR THEIR PROPER IMPACT ON SOCIETY: WHAT TOOLS ARE AVAILABLE?}

Copyright: (C) 2021 CSIC. Este es un artículo de acceso abierto distribuido bajo los términos de la licencia de uso y distribución Creative Commons Reconocimiento 4.0 Internacional (CC BY 4.0).

ABSTRACT: Artificial Intelligence $(\mathrm{Al})$ and robotics are two realities that are here to stay and whose involvement in our society covers a multitude of very diverse areas. The fact that we are dealing with a technology capable of emulating human attitudes, such as learning or adaptation, makes Al, and specifically robots equipped with it, unpredictable machines. Furthermore, the potential interaction between machines and vulnerable subjects, such as consumers or patients, is a compounding factor, which implies even greater risk. This is precisely why it is necessary to analyse the legal and ethical consequences of the use of intelligent robots in our society.

KEYWORDS: Robotics, Artificial Intelligence, liability, data protection, regulation, COVID-19. 


\section{INTRODUCCIÓN: PRINCIPALES RETOS E INTE- RROGANTES JURÍDICOS DE LA INCLUSIÓN DE RO- BOTS INTELIGENTES}

La robótica y todo lo que este nuevo ámbito supone plantea nuevos retos en materia de seguridad y garantías que deben prestarse a los usuarios de productos que se enmarcan en dicho sector.

En efecto, desde el punto de vista de la regulación empresarial, los robots y la inteligencia artificial en general, supondrán un esfuerzo legislativo y un reto para realizar una aplicación adecuada del Derecho. Habrá que estudiar si la legislación actual aplicable en los Estados Miembros da cobertura y es suficiente para hacer frente a los riesgos y nuevas situaciones y hechos que puedan producirse cuando este tipo de productos se extienda en el mercado y sean utilizados por personas físicas o jurídicas, públicas o privadas.

Por otra parte, debe contemplarse las formas de administrar y gestionar las empresas que utilicen este tipo de productos tanto en su producción como en su organización. En este sentido merecen una especial atención las posibles responsabilidades del empresario, tanto en el ámbito civil como penal e, incluso, en el ámbito de la responsabilidad administrativa, así como la Responsabilidad Social Corporativa, entendida ésta como la forma de administrar y gestionar las nuevas situaciones que se planteen, así como los códigos éticos que deban formalizarse en dichas empresas.

Los juristas deberán también valorar si el ordenamiento jurídico español, en particular, y el europeo en general, están preparados para resolver la problemática mercantil-empresarial que puedan generar los robots; especialmente, aquéllos que interactúen de manera física y cognitiva con humanos.

En ese sentido deberían estudiarse los problemas de responsabilidad por daños que puedan producirse en los usuarios, así como en otros bienes que recaerían, en principio, en el fabricante o el importador del robot. En la actualidad en España esto está previsto en el Real Decreto Legislativo 1/2007 de 16 de noviembre, por el que se aprueba el Texto Refundido de la Ley General para la Defensa de los Consumidores y Usuarios (en adelante LGDCU ) y otras leyes complementarias, respecto a los Productos Defectuosos y en el Código Civil respecto a la responsabilidad contractual y extracontractual, así como en las normas relativas a la protección de datos.
Así mismo, habrá que estudiar si las actuales normas relativas a los seguros, en concreto la Ley 50/1980 de 8 de octubre de Contrato de Seguro, ofrecen una cobertura adecuada a la problemática que plantearán los riesgos que generen este tipo de productos, que probablemente obligarán a las compañías de seguros a adecuarse a las nuevas circunstancias y a prever nuevas circunstancias en las pólizas.

Por otro lado, la robótica y la inteligencia artificial, van adquiriendo un protagonismo cada vez mayor en la gestión de sectores empresariales como el bancario, el de los seguros y el del mercado de valores, y en otros ámbitos de carácter público o privado como la administración pública, la sanidad, la seguridad del Estado, la cultura, la educación. Hoy, en todos estos ámbitos, es indispensable la incorporación de datos de diferente carácter y con distinta intención, en ocasiones sensibles para la intimidad de las personas y para la toma de decisiones de cualquier naturaleza.

Por ello, es necesario que exista un control en la introducción de los datos, que no sólo los verifique, también supervise su privacidad y los proteja. Este control debe también velar por un uso pertinente, de los datos, su ecuanimidad y no discriminación de los derechos humanos, sociales y políticos.

Por otro lado, el manejo de datos como función o cualidad de los robots, podría constituir un factor crucial a la hora de establecer diferentes categorías o tipos de robots, al tratarse de un concepto bastante amplio e, incluso, indeterminado. Según la Real Academia Española, robot se define como «máquina programable capaz de realizar trabajos antes reservados sólo a las personas».

Esta definición es tan amplia, que abarca desde los electrodomésticos hasta complejos y sofisticados instrumentos cuya actividad es aún impredecible. Por tanto, para poder analizar la eficacia de las diferentes herramientas jurídicas disponibles es necesario establecer una línea distintiva, y esa línea es sin duda, la presencia o no de inteligencia artificial en su composición.

Al respecto, cabría plantearse entonces, ya no sólo si son eficaces las normas acerca de la responsabilidad y los seguros, sino también si las largas y, teóricamente, exigentes y pormenorizadas normas sobre la protección de datos existentes en Europa, están cumpliendo su función.

Para llevar a cabo este análisis es necesario enfocar la figura del robot desde las dos perspectivas anterior- 
mente anunciadas: como una máquina desprovista de cualquier tipo de sofisticación en forma de Inteligencia Artificial, y cómo artefacto dotado ciertas capacidades humanas.

En este sentido algunos autores no tienen en cuenta dentro de la definición de robot strictu senso a aquellas máquinas que deban ser dirigidas por un humano o que funcionen de forma semiautomática, quedando únicamente incluidos aquellos capaces de procesar información y actuar en función de esta (Anguita Ríos, 2020: 2256). En cualquier caso, bajo nuestro punto de vista, un robot sencillo -que no cuenta con ningún tipo de inteligencia como puede ser un juguete o un electrodoméstico no inteligente-, no presenta en principio, riesgos a ningún efecto de los que se pretenden analizar aquí; en el sentido de que nuestras normas jurídicas ya predicen fórmulas claras para solucionar los problemas que tienen que ver con este tipo de tecnología. De hecho, los esfuerzos de la Unión Europea en el tema de la robótica y la Inteligencia Artificial se han centrado, entre otros aspectos, en la búsqueda de elementos que ayuden a discernir qué se considera un robot inteligente (Parlamento Europeo, 2020).

En cualquier caso, la problemática que gira en torno a la robótica desde el punto de vista jurídico, a nuestro juicio, estriba en la idea de que los robots sean calificados o no, como máquinas capaces de aprender y de tomar decisiones con cierta autonomía. En otras palabras, podríamos decir que aquellos robots que puedan equipararse a un bien inmueble como una máquina (art. 335.4 del Código Civil (CC)) es probable que puedan encajarse más fácilmente en la normativa ya establecida, en contraposición a los robots dotados de IA, supuesto nos enfrenta al reto de la imprevisibilidad en los comportamientos de éstos últimos.

Por ello cuanto mayor sea la autonomía con la que actúan los robots, mayor será la dificultad para establecer las distintas responsabilidades en las que pueda incurrirse, lo cual dificulta sobremanera la posibilidad de asociar las distintas categorías de robots a alguna de las categorías preexistentes y sus correspondientes relaciones jurídicas que han sido establecidas en nuestro Derecho (Anguita Ríos, 2020: 2259).

\section{LOS ROBOTS Y LOS CONSUMIDORES}

\subsection{La protección jurídica actual y sus posibles efectos}

La imprevisibilidad a la que se ha hecho referencia presenta una doble vertiente. La primera se encuen- tra estrechamente relacionada con el procesamiento y el uso que se dé a la gran cantidad de datos que puede llegar a manejarse en la robótica; y la segunda se refiere a la posibilidad de que los denominados robots pueden llegar a provocar daños físicos.

Por otra parte, debe considerarse también el ámbito de aplicación al que pertenecen los distintos tipos de robots. En este sentido, y según la Strategic Research Agenda for Robotics in Europe 2020-2021, los sectores en los que la robótica tiene un mayor impacto son de diversa naturaleza. Entre ellos deben destacarse las manufacturas, la medicina, la agricultura, la logística y el transporte y el consumo empresarial (en inglés business to business o $B 2 B$ ) y doméstico (en inglés business to consumers o B2C) (Comisión Europea, 2021).

La razón por la que debe realizarse una distinción es porque existen diferentes niveles de protección en nuestro derecho, atendiendo principalmente a las partes implicadas en los supuestos de hecho que se planteen en cada caso. En este sentido, una de las figuras más protegidos por el legislador es la del consumidor, entendido éste como toda «persona física o jurídica que actúa en un ámbito ajeno a una actividad empresarial o profesional» (LGDCU).

A este respecto, puede señalarse como ejemplo las medidas especiales que se contemplan en sectores tales como el bancario y ello con el fin de otorgar una mayor protección al consumidor respecto a sus productos o servicios.

En efecto, en el sector bancario además de tener una legislación exigente en cuanto al cumplimiento de normas especiales sobre contabilidad, seguridad y estabilidad económica, se ha establecido un sistema de supervisión ejercido por los Bancos Nacionales correspondientes -el Banco de España en el caso español-, y el ejercido por el Banco Central Europeo en su ámbito de competencia.

En ese sentido, debe decirse que en la actualidad el sector de la robótica carece de la trascendencia que tienen los bancos para la estabilidad y seguridad económica de un país. Los bancos suponen un elemento esencial de la economía de un país porque gestionan los ahorros de los ciudadanos, financian a empresarios y particulares, y a los propios Estados.

En ese sentido la labor y la función de la robótica hoy no cumple el carácter fundamental que realizan las entidades bancarias; pero no podemos negar, sin embargo, que sus implicaciones actuales alcanzan 
niveles muy significativos en lo que atañe a sus relaciones con los consumidores y que lo serán aún más en el futuro. El ejemplo del sector bancario nos sirve para entender cómo los consumidores y usuarios son sujetos a los que se reviste de una especial protección por su situación de vulnerabilidad, frente a otras personas con un mayor poder económico o protegidos por otras formas jurídicas.

Al respecto, debe decirse que el mercado de los consumidores constituye un ámbito fundamental del desarrollo de la robótica, existiendo multitud de robots inteligentes y no inteligentes, que se venden como bienes de consumo, lo que hace que sean los datos de aquéllos los que se vean comprometidos en mayor medida y expuestos a ciberataques (Parlamento Europeo, 2019).

En ese mismo sentido, puede observarse que algunos consumidores se encuentran bajo el paraguas de una normativa aún más garantista, como, por ejemplo, los menores de edad que se ven amparados por normas especiales en materia de protección de datos personales (art. 8 del Reglamento General de Protección de Datos (RGPD)).

Por todo ello, debe ponerse especial atención en la situación de los consumidores en materia de protección de sus derechos al adquirir bienes en el ámbito de la robótica, tanto en lo que tiene que ver con la seguridad física como en relación a los datos que se utilicen.

Por otro lado, y dejando a un lado el ámbito del consumo, debe destacarse otra área en la cual el impacto de la robótica en las personas físicas es directo y de gran importancia: la medicina.

En efecto los robots comienzan a jugar un papel muy importante tanto en los tratamientos como en las intervenciones médicas. Existen robots que participan en procesos de cirugía, de diagnóstico y de rehabilitación; incluso podríamos plantearnos su conveniencia a la hora de trabajar con pacientes con COVID-19. En este sentido los robots, aunque estén dotados de estructuras corpóreas, muchas veces similares a las humanas, no dejan de ser máquinas y, por tanto, no se ven amenazados por enfermedades o, como en este caso, por un virus. Por ello, su presencia en lo que tiene que ver con la asistencia de pacientes cuyo tratamiento arriesga, como hemos visto de manera importante, la vida de los trabajadores sanitarios, es una idea interesante. En este caso, se plantearían cuestiones similares a las que hemos visto al hablar de los consumidores, si bien con diferencias que deban analizarse y realizarse en cada caso.

\subsection{Principales problemas y soluciones a la responsa- bilidad por daños producidos por robots}

La responsabilidad por daños plantea dos problemas de singular importancia. El primero, como ya se ha mencionado, se refiere al comportamiento inesperado que podrían llegar a manifestar los robots; y el segundo, a la variedad y número de actores que están presentes en los procesos de funcionamiento de un robot (Lacruz Mantecón, 2019: 2378). En este sentido, deben destacarse: los diseñadores, los fabricantes, y los propios usuarios que los incorporan a sus vidas y lo mismo puede decirse respecto al sector sanitario, donde actúan el médico o profesional sanitario, el fabricante de la solución tecnológica y la institución sanitaria que ofrece el tratamiento (Parlamento Europeo, 2019).

La intervención de distintos sujetos en los ámbitos señalados, dificultan la aplicación de la normativa que regula la responsabilidad extracontractual, entendiéndose ésta como aquélla que responde de los daños producidos de manera independiente de cualquier relación jurídica preexistente entre las partes.

La LGDCU constriñe la responsabilidad por daños causados por productos defectuosos a la figura del productor (art.135, LGDCU). Además, la misma norma define producto defectuoso como «aquél que no ofrezca la seguridad que cabría legítimamente espe$\operatorname{rar}(. .) ».($ art.137, LGDCU). Dicha responsabilidad supone que en caso de que medie culpa o negligencia por parte del productor, éste debe ser quien responda del daño producido, siempre que se pruebe la relación de causalidad directa entre el defecto y el daño, correspondiéndole esta tarea al perjudicado en cuestión (art. 139 LGDCU).

Las razones que explican las dificultades existentes ante esta normativa responden a varios motivos:

1. En primer lugar, ¿podría definirse como defectuoso un robot que ha llevado a cabo un acto de carácter lesivo, en algunos casos imprevisibles, y que se fundamenta en un aprendizaje autónomo que está fuera de lo que, en un primer momento, estableció el propio fabricante o desarrollador del programa? Debe señalarse que ese aprendizaje se genera a partir del momento en el que comienza a utilizarse. Se habla de la seguridad que cabe esperarse legítima- 
mente, pero en este caso ¿podría escaparse de las previsiones humanas el control de los actos del robot? (Lacruz Mantecón, 2019: 2378) Y lo que es más importante ¿de la responsabilidad del fabricante o programador?

2. En el caso de robots inteligentes, es muy complicado determinar si la razón de su comportamiento es un fallo en alguno de los componentes de fabricación del programa con el que ha sido diseñado, del uso que se haya hecho de él, del programa con el que funciona, o si se debe a una acción autónoma del propio robot basada en el aprendizaje previo de su entorno (Anguita Ríos, 2020: 2559 y ss.). Por tanto, prima facie, no parece del todo correcto considerar al fabricante como responsable de los daños causados por el robot.

3. Los perjudicados deben demostrar una relación de causalidad que, en este caso, por tratarse de máquinas de gran complejidad, se trataría de una tarea muy difícil.

Esta problemática responde a la falta de acogimiento por parte de las normativas de la nueva realidad tecnológica que rompe con fuerza dentro de los distintos sectores productivos, por lo que es evidente que necesitamos nuevos instrumentos que sean capaces de acoger estos cambios y que sirvan como complemento a los ya existentes. Hoy en día, ya se han formulado diferentes posibilidades que en un futuro podrían quedar recogidas en nuestras leyes.

En primer lugar, considerar que sea el propio titular del robot el que tenga que responder de los daños causados a terceros, estableciéndose así una responsabilidad del mismo. Sin embargo, ello pasaría por dotar de un seguro obligatorio al robot, como ocurre con los vehículos, de manera que se cuente con un fondo que pueda proteger a las víctimas de las actuaciones independientes de estas máquinas (Lacruz Mantecón, 2019: 2379). Este sistema pasaría por establecer un registro de los propios robots a los que se les asignaría una especie de matrícula con un número de póliza (Anguita Ríos, 2020: 25561 y ss.), con lo que habría así un mayor control de los robots y se conseguiría una mayor seguridad jurídica para consumidores y fabricantes, e incluso una mayor aceptación social.

En el mismo sentido, y siguiendo la misma línea de razonamiento, se podría dotar de un seguro obligatorio a los propios fabricantes de robots inteligentes
(Anguita Ríos, 2020: 2570). De esta forma se eliminarían barreras disuasorias a la producción de máquinas inteligentes a la vez que se aseguraría la existencia de un fondo que pudiera resarcir a las víctimas.

Una propuesta más disruptiva y cargada de controversia es la de dotar de personalidad jurídica al robot, de manera que se constituya como un sujeto de obligaciones que pueda responder de los daños provocados por su acción. En tal caso se podría suplir su falta de patrimonio creando un fondo que nutrirían de manera conjunta, los diseñadores, fabricantes y usuarios (Comité Económico y Social Europeo, 2017), a través, por ejemplo, del pago de una cantidad extra a la hora de adquirir el producto (Anguita Ríos, 2020: 2560).

Esta primera idea vio la luz en la Resolución del Parlamento Europeo de 16 de febrero de 2017, con recomendaciones destinadas a la Comisión sobre normas de Derecho civil sobre robótica, al plantear el término «personalidad electrónica» como una posible alternativa en el futuro para aquellos robots más complejos (apdo. 59.f).

Sin embargo, el trasfondo de esta idea se encuentra plagada de interrogantes y de dificultades ya no sólo legales, sino sobre todo, a nuestro juicio, de carácter ético y moral. En esta línea se han manifestado por ejemplo el Comité Económico y Social Europeo (2017: 2) en uno de sus dictámenes titulado Inteligencia artificial: las consecuencias de la inteligencia artificial para el mercado único (digital), la producción, el consumo, el empleo y la sociedad, dónde cuestiona no sólo las implicaciones éticas y morales sino que argumenta también que «podría desaparecer el efecto correctivo y preventivo de la responsabilidad civil si esta dejase de recaer sobre el autor por haberse transferido al robot». Añade, además, que una forma jurídica como la que se plantea podría ser «susceptible de uso y aplicación indebidos».

Por otra parte, debe tenerse en cuenta también que la atribución de personalidad jurídica a los robots, aunque pueda resolver el problema de la responsabilidad, desplazándola al propio robot, exige revestir a un sujeto, no sólo de obligaciones, sino también de derechos, es decir, de los derechos de los que gozan las personas físicas y jurídicas. Lo cual, a nuestro juicio, carece de rigor jurídico y, al menos, a día de hoy no tiene amparo en nuestro ordenamiento.

En su momento los miembros del Parlamento Europeo a favor de una resolución en ese sentido, hablaban de un tipo de personalidad que permitiera al 
robot, al igual que las organizaciones con personalidad jurídica, poder llevar a cabo actos como la firma de contratos o la capacidad para ser demandados. La entidad de esta personalidad jurídica, sin embargo, no llegaría a situarse al mismo nivel que las existentes, en el sentido de que lo que se buscaría es hacer al robot sujeto de obligaciones, por ejemplo, a través de un seguro obligatorio, como el que avanzábamos, pero limitando, a su vez, sus derechos (Delcker, 11 de abril de 2018).

Sin embargo, el rumbo que parece estar tomando la normativa en materia de IA, en cualquiera de sus aplicaciones, coloca como pilar fundamental la supervisión humana.

En efecto, la IA no debe en ningún momento «disminuir, limitar o desorientar la autonomía humana». Para ello, es necesario que los sistemas de IA promuevan una sociedad democrática, y equitativa, complementen y mejoren la acción humana y el respeto a los derechos fundamentales (Grupo Independiente de Expertos de Alto nivel sobre IA, 2018: 18).

\section{EN BUSCA DE UNA INTELIGENCIA ARTIFICIAL FIABLE}

\subsection{Limitaciones a la discrecionalidad de los robots y manejo de principios éticos}

Una vez aclarado que la independencia y libertad humanas deben situarse como pilares fundamentales en el diseño de la actuación de los robots inteligentes, habría que preguntarse de qué manera se podría configurar un sistema de supervisión por parte de las personas, que fuera lo suficientemente garantista pero que, al tiempo respetara y fomentara la innovación propia de esta tecnología.

No sería algo factible un tipo de participación humana que implicara una intervención por parte de las personas de todos los procesos de decisión de la IA; pero sí podría darse cierta discrecionalidad a las mismas durante la utilización de los sistemas de IA. Al respecto, podría asemejarse al poder decidir sobre la aceptación o no de una determinada actuación que vaya a llevar a cabo el sistema (Grupo Independiente de Expertos de Alto nivel sobre IA, 2018:19). En este sentido se podrían establecer los niveles de discrecionalidad dependiendo de los campos en lo que se implante.

De esta manera debería valorarse cuando se va a implantar un programa informático a una estructura corpórea como un robot, si va a estar en contacto con personas vulnerables como niños o ancianos. En este último caso, los niveles de control deberían poder ser mayores por los riesgos incrementales que implica el uso de robots inteligentes de manera general, y de manera especial, cuando se refiere a la relación que puedan tener los robots con este tipo de sujetos. Como ejemplo de posibles medidas a tomar, podrían ser la capacidad de poder desconectar al robot en caso de emergencia (Anguita Ríos, 2020: 2550), o la existencia de un control parental en caso de los menores.

Por todo ello puede concluirse que, para poder llevar a cabo una correcta supervisión de los robots, es necesario tener un mayor conocimiento de la IA, que debe implicar a toda la sociedad en su conjunto, aunque de manera especial, a las partes implicadas directamente en el ciclo de vida de los robots inteligentes: los diseñadores, desarrolladores, fabricantes y usuarios finales de los productos (Grupo Independiente de Expertos de Alto nivel sobre IA, 2018: 8).

En este sentido, es necesario establecer una serie de principios éticos universales que sean conocidos por todas las partes que se introduzcan y apliquen en los procesos de diseño de la IA, que establezcan mecanismos de comprobación del correcto cumplimiento de estos requisitos a la hora de ofrecer robots dotados de IA y, finalmente, que los propios usuarios puedan reclamar el cumplimiento de esos requisitos en cada caso.

Estos principios no constituyen una mera idea lanzada al aire, sino que ya han sido estudiados y establecidos en documentos oficiales. Como ejemplo la lista de requisitos éticos promulgados por el Grupo Independiente de Expertos de Alto nivel sobre IA de la Comisión que incluye «el respeto de la autonomía humana» y la "prevención del daño; equidad; explicabilidad» (Grupo Independiente de Expertos de Alto nivel sobre IA, 2018:14). Aunque no se trate de un texto de derecho positivo y tan solo sean orientaciones para los profesionales de la IA, los principios señalados encuentran su fuente de inspiración en los derechos fundamentales, recogidos en mayor o menor medida en textos como el RGPD o la LGDCU.

Por tanto, estos principios pueden infundir un nuevo sentido a la aplicación de los textos ya existentes en temas focalizados en la IA, contribuyendo así a una interpretación más fiel de nuestro nuevo entorno, pudiendo ser una fuente de inspiración, una ne- 
cesaria normativa, que algunos ya llaman de forma globalizada "Derecho de los robots» (Barrio Andrés, 2018). Precisamente, y dada la inseguridad jurídica que puede llegar a generar como ya se ha visto al hablar de la responsabilidad por daños, es de urgente necesidad lograr una normativa común que recoja unos principios éticos que sirvan de base para el despliegue y manejo de la IA y la robótica, (Parlamento Europeo, 2020).

\subsection{La protección de datos}

Una de las ventajas de la existencia de unos principios éticos comunes sería como se ha indicado antes un aumento de la información disponible acerca de lo que debería ser la IA fiable, lo que ayudaría a un mayor acogimiento de ésta en nuestra sociedad y un mayor empoderamiento de los consumidores, en lo que tiene que ver con la responsabilidad y con la protección de sus datos. Este último punto es de especial relevancia tal y como hemos dicho, ya que los robots dotados de IA recopilan y etiquetan datos del entorno en el que se encuentran, y en mayor o menor medida, aprenden de los mismos. Asimismo, el propio diseño de los robots inteligentes implica la implantación de una serie de algoritmos y datos que variarán dependiendo del uso para el que vaya a ser destinados en un futuro. Debido a este uso masivo de datos debería revisarse, por un lado, la validez del corpus legislativo existente en materia de protección de la privacidad, como son el RGPD o el próximo Reglamento sobre la privacidad y las comunicaciones electrónicas ${ }^{1}$. Muchos dudan de su eficacia, y se preguntan si los robots y la IA van a ofrecer las debidas garantías, teniendo en cuenta que los pequeños artilugios que utilizamos a diario en nuestras vidas profesionales y personales nos sorprenden, demasiado a menudo, con mensajes que nos hacen dudar de nuestra privacidad ${ }^{2}$. Es por ello, que desde el Parlamento Europeo se afirma que «puede resultar necesario adaptar hasta cierto punto algunos instrumentos jurídicos concretos al objeto de reflejar la transformación digital y dar respuesta a los nuevos retos que plantea el uso de la inteligencia artificial» (Parlamento Europeo, 2020: 5).
Por otro lado, será necesario contar con mecanismos que permitan evaluar todo lo que tiene que ver con el proceso de diseño de los robots, en concreto con el posible impacto de los diferentes datos que se le implantan. De hecho, esto último ya se recoge también dentro de las propuestas del Comité de Expertos al hablar de la auditabilidad de la IA (Grupo Independiente de Expertos de Alto nivel sobre IA, 2018: 24), y además se asemeja a herramientas utilizadas ya en otros sectores. Un ejemplo serían las pruebas de stress test de los bancos en las que se realizan simulaciones de diferentes escenarios económicos para conocer la situación hipotética en la que se encontrarían los bancos al funcionar en esas circunstancias. De esta manera se podrían diseñar robots desde la una perspectiva preventiva en lo que tiene que ver con los riesgos, minimizándolos en la medida de posible. En este sentido no hay que olvidar la repercusión del entorno y de la interacción con los diferentes agentes en lo que se refiere a la actuación posterior y más cuando hablamos de sujetos que buscan relacionarse de forma contenciosa con estas máquinas. Por ello, sería necesario o bien dotar a los robots de mecanismos de discriminación de información, teniendo de base los principios éticos que se comentaban, o bien incrementar los controles para la adquisición de este tipo de máquinas por parte de los consumidores y las empresas, de manera que se garantice la idoneidad de los diferentes sujetos y entidades que vayan a manejar estos robots.

\subsection{El futuro de la regulación}

Las últimas tendencias normativas parecen inclinarse más por un control sobre los sistemas de IA, y así lo demuestran las nuevas líneas de actuación recogidas dentro del marco reglamentario propuesto desde las instituciones europeas. En 2020 el Parlamento y el Consejo europeos aprobaron una resolución que contenía una propuesta legislativa de Reglamento sobre los principios éticos de la IA y la robótica. Por ejemplo, el legislador proponía llevar a cabo una «evaluación de riesgos $»^{3}$ de estas tecnologías bajo unos criterios objetivos, así como, en caso de considerarse de alto

1 Propuesta de Reglamento del Parlamento Europeo y del Consejo sobre el respeto de la vida privada y la protección de los datos personales en el sector de las comunicaciones electrónicas y por el que se deroga la Directiva 2002/58/CE (Reglamento sobre la privacidad y las comunicaciones electrónicas). Disponible en https://eur-lex.europa.eu/legal-content/ES/TXT/?uri=CELEX\%3A52017PC0010 [Fecha de consulta: 23 noviembre 2021].

2 La CIA desclasifica documentos sobre un robot mosquito espía. Disponible en: https://mundo.sputniknews.com/seguridad/202002191090529199-la-cia-desclasifica-documentos-sobre-un-robot-insecto-espia--video/ [Fecha de consulta: 23 noviembre 2021].

3 Dependiendo de la función que desempeñe la IA se diferencian la IA de riesgo inaceptable, alto, limitado y reducido (Comisión Europea, 2021a) [Fecha de consulta: 23 de noviembre de 2021]. 
riesgo ${ }^{4}$, una "evaluación de conformidad» con los requisitos éticos establecidos en el propio texto de la propuesta de Reglamento. Esto último daría lugar a la expedición del llamado «Certificado europeo de calidad ética» en caso de arrojar un resultado positivo. Además, se establecería la figura de una «autoridad de control» en cada Estado Miembro para garantizar la aplicación del Reglamento, y efectuar las evaluaciones en cuestión (arts. 14,15,16,17,18 de la Propuesta de Reglamento del Parlamento Europeo y del Consejo por el que se establecen normas armonizadas en materia de Inteligencia Artificial (ley de inteligencia artificial). Posteriormente el 24 de abril de 2021 la Comisión publicó otra propuesta de Reglamento que recogía la llamada Ley de Inteligencia Artificial, desarrollando las ideas anteriormente expuestas y ampliando la regulación sobre la introducción al mercado y el manejo de la IA. En este sentido, la IA que se encuentra fuertemente regulada es la considerada de alto riesgo ${ }^{5}$, dedicándose a esta la mayor parte de su articulado. La normativa establece de forma específica una serie de líneas rojas a los usos de la misma, así como fuertes obligaciones relacionadas con la supervisión humana, la adecuada gestión de riesgos, la calidad del dato o la transparencia (Comisión Europea, 2021a; Anguita Ríos, 2020: 3-5). Por otra parte, los proveedores y usuarios de tecnologías consideradas de riesgo limitado o reducido ${ }^{6}$ únicamente deberán cumplir con medidas de transparencia y manejo respectivamente, quedando fuera de la regulación las categorizadas de riesgo mínimo o nulo (Anguita Ríos, 2020: 5).

De esta manera, el nuevo marco regulatorio pretende garantizar la seguridad de estas tecnologías durante todo su ciclo de vida, imponiendo medidas de hard law o soft law en función del riesgo que tengan asociado. Al mismo tiempo, se pretenda impulsar con esta legislación el desarrollo y despliegue de estas tecnologías, para lo cual se dedicarían por ejemplo recursos en investigación o se llevarían a cabo consultas públicas sobre los proyectos que se impulsen en esta área (Comisión Europea, 2021b).

\section{CONCLUSIÓN}

Una vez planteados los principales problemas jurídicos y éticos que se desprenden del uso de los robots inteligentes, es necesario reflexionar sobre la necesidad de dotar de unas garantías lo suficientemente altas a los principales sujetos implicados, de manera que se consiga preservar la seguridad jurídica y la innovación. Es cierto que es un equilibrio nada fácil de conseguir, pero sin duda ejemplos como la creación de un Código Ético en aras de lograr una IA fiable, o los estudios de la cuestión por comités de expertos ligados a las fuerzas legislativas, son sin duda iniciativas que trazan el camino correcto hacia una regulación proactiva, más que reactiva, de los robots y en general de la IA, en nuestra sociedad. Hace falta la colaboración entre empresas, ciudadanos y entes legislativos, empleando para ello instrumentos como consultas públicas que ayuden a concretar una senda adaptada a las múltiples necesidades de los distintos sujetos intervinientes en el ciclo de vida de los robots, y, a su vez, a alentar la creación de nuevos proyectos que impliquen a estas tecnologías, especialmente en aquellas áreas donde pueden generar un gran valor añadido. En este sentido, sería muy positivo reflexionar acerca de la conveniencia de proponer proyectos relacionados con la robotización y la implantación de sistemas de IA, como parte del Plan de Recuperación y Resiliencia tras el COVID-19. Más aún si cabe, si tenemos en cuenta como se ha indicado, las ventajas que los robots inteligentes pueden ofrecer en lo que tiene que ver con la atención a pacientes de enfermedades infecciosas.

Por otro lado, las propuestas que se contemplan dentro de las nuevas líneas normativas expuestas parecen arrojar un poco más de luz sobre cómo será la regulación de estas tecnologías. Tal y como se des-

4 Por ejemplo: «el software para la clasificación de los currículos en los procesos de selección de personal, la aplicación de la IA en la cirugía asistida por robots, la calificación de exámenes etc.» (Comisión Europea, 2021a) [Fecha de consulta: 23 de noviembre de 2021].

5 Se entiende por alto riesgo: «riesgo significativo, derivado del desarrollo, el despliegue y el uso de la inteligencia artificial, la robótica y las tecnologías conexas, de causar lesiones o daños a las personas o a la sociedad, vulnerando los derechos fundamentales y las normas de seguridad establecidas en el Derecho de la Unión, teniendo en cuenta su uso o finalidad específicos, el sector en el que se desarrollan, despliegan o usan y la gravedad de las lesiones o daños que cabe esperar que se produzcan» (art.4 de la propuesta de Reglamento del Parlamento Europeo y del Consejo sobre los principios éticos para el desarrollo, el despliegue y el uso de la inteligencia artificial, la robótica y las tecnologías conexa) [Fecha de consulta: 23 de noviembre de 2021]. Ver en: https://www.europarl.europa.eu/doceo/ document/TA-9-2020-0275_ES.html\#title17

6 «Al utilizar sistemas de IA como robots conversacionales, los usuarios deberán ser conscientes de que están interactuando con una máquina para poder tomar una decisión informada de continuar o no» (Comisión Europea, 2021b) [Fecha de consulta: 23 de noviembre de 2021]. 
prende de los principios antes enunciados, vemos que la perspectiva antropocéntrica sigue siendo una constante en la fórmula regulatoria de las autoridades de la Unión Europea con respecto a este tema y sin duda sigue la línea de lo que venimos exponiendo a lo largo de todo nuestro análisis, como es la necesidad de una supervisión humana en todo el ciclo de vida de estas tecnologías, lo cual no es incompatible con el pleno desarrollo tecnológico, sino que colabora en el desarrollo de una IA responsable, segura y fiable para todos. Por otro lado, la importancia de estas propuestas radica también en la necesidad existente de constituir un marco normativo único y armonizado, en el que exista una colaboración entre los Estados Miembros bajo unos principios éticos compartidos, habida cuenta de las ventajas que esto supondría para el buen funcionamiento del mercado único digital y la correcta explotación de estas tecnologías. No obstante, hay que tener en cuenta dos cuestiones importantes. La primera es que a pesar de tratarse de una regulación novedosa y que podría servir de modelo para otras legislaciones (como ocurrió en su momento con el RGPD) esta debe estar lo suficientemente bien diseñada para no suponer una traba al desarrollo de la IA. Precisamente un sistema como este, en el que existan herramientas que limiten en cierta medida su manejo, que impongan trámites administrativos para poder comercializar con ciertas tecnologías y con un sistema de multas adherido, corre el riesgo si no se implementa con la eficiencia y agilidad suficiente, de poner muros al desarrollo, actuando así en detrimento de las empresas europeas frente a otros líderes internacionales (de Miguel Asensio, 2021: 3). No obstante, vemos como a la mayor parte de la IA existente que se corresponde con aquella de riesgo limitado, mínimo y nulo, no le aplican estas medidas. En segundo lugar, a pesar de que se analizan cuestiones de gran enjundia que rodean a la IA y tecnologías conexas, no se aborda la cuestión de la responsabilidad en los términos que hemos analizado, por lo que habrá que estar a las novedades que vayan surgiendo en este ámbito. En este sentido, la Comisión ya ha anunciado que se revisará la Directiva sobre responsabilidad por daños de manera que incluya las novedades normativas referentes a la IA y otras tecnologías (Anguita Ríos, 2020: 9).

Para concluir, esperamos que las máquinas que hemos creado a nuestra imagen y semejanza, aunque desplacen ciertas habilidades humanas, nunca lleguen a desplazar las particularidades de la persona humana, sino que seamos nosotros los que establezcamos siempre las reglas y los límites respetando nuestra propia singularidad, haciendo de los robots un instrumento de ayuda y de los que nos podamos servir. Por último, en ningún caso debe destruir, ni desplazar, lo mejor de nuestra forma de vida, en definitiva, lo mejor de nosotros mismos.

\section{AGRADECIMIENTOS:}

Este trabajo se ha realizado en el marco del proyecto de investigación INBOTS (H2020, ref. 780073). Quiero agradecer a Dña. Natalia Zardoya el magnífico trabajo que ha realizado para la Revista Arbor, su rigor jurídico, su pundonor y su paciencia (Christi Amesti). Quiero agradecer a Dña. Christi Amesti por su confianza, apoyo y cariño durante la elaboración de este artículo (Natalia Zardoya).

\section{REFERENCIAS}

Anguita Ríos, Rosa María (2020). Inteligencia Artificial y Derecho Civil: líneas de pensamiento en materia de daños. Revista Crítica de Derecho Inmobiliario (781): 2541-2581

Barrio Andrés, Moisés (dir.) (2018). Derecho de los robots. Wolters Kluwer.

Comisión Europea. (2021a). Excelencia y confianza en la inteligencia artificial. Disponible en https://ec.europa.eu/ info/strategy/priorities-2019-2024/europe-fit-digital-age/excellence-trust-artificial-intelligence_es

Comisión Europea (2021b). Reglamento del Parlamento Europeo y del Consejo por el que se establecen normas armo- nizadas en materia de inteligencia artificial (ley de inteligencia artificial) y se modifican determinados actos legislativos de la Unión. Disponible en: https:// eur-lex.europa.eu/legal-content/ES/ TXT/?uri=CELEX:52021PC0206

Comité Económico y Social Europeo (2017). Inteligencia artificial: las consecuencias de la inteligencia artificial para el mercado único (digital), la producción, el consumo, el empleo y la sociedad. Dictamen de iniciativa. Disponible en https://eur-lex.europa.eu/legal-content/ ES/ALL/?uri=CELEX\%3A52016IE5369

Delcker, Janosch (11 de abril de 2018). Europe divided over robot 'personhood'. POLITICO. Disponible en https:// www.politico.eu/article/europe-divi-

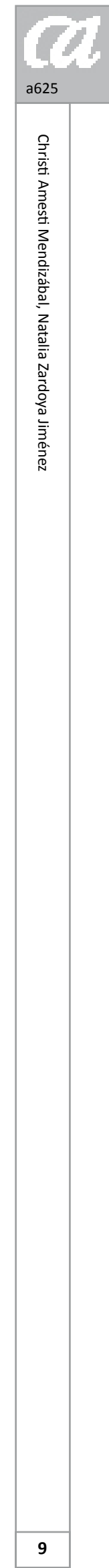

ded-over-robot-ai-artificial-intelligence-personhood/

Grupo Independiente de Expertos de Alto nivel sobre IA. (2018). Directrices éticas para una IA fiable. Comisión Europea. Disponible en https://ec.europa.eu/ newsroom/dae/document.cfm?doc_ id $=60423$

Lacruz Mantecón, Miguel I. (2019). Inteligencia Artificial y coches autónomos. Análisis jurídicos europeos. Revista Crítica de Derecho Inmobiliario, 95 (775): 2373-2409.

Miguel Asensio, Pedro Alberto de (2021). Propuesta de Reglamento sobre Inteligencia Artificial. La ley Unión Europea, 92, 1-8. 
Parlamento Europeo. (2019). Una política industrial global europea en materia de inteligencia artificial y robótica. Resolución, Estrasburgo. Disponible en https://www.europarl.europa.eu/ doceo/document/TA-8-2019-0081 ES.pdf
Parlamento Europeo (2020). Resolución del Parlamento Europeo, de 20 de octubre de 2020, con recomendaciones destinadas a la Comisión sobre un marco de los aspectos éticos de la inteligencia artificial, la robótica y las tecnologías conexas que contiene la Propuesta de
Reglamento del Parlamento Europeo y del Consejo por el que se establecen normas armonizadas en materia de Inteligencia Artificial (ley de inteligencia artificial). Disponible en: https://www. europarl.europa.eu/doceo/document/ TA-9-2020-0275_ES.html\#title1 